\title{
The detection of tethered and rising bubbles using multiple acoustic techniques
}

\author{
Timothy G. Leighton, David G. Ramble, and Andy D. Phelps \\ Institute of Sound and Vibration Research, University of Southampton, Highfield, Southampton SO17 1BJ, \\ United Kingdom
}

(Received 22 January 1996; accepted for publication 10 November 1996)

\begin{abstract}
There exists a range of acoustic techniques for characterizing bubble populations within liquids. Each technique has limitations, and complete characterization of a population requires the sequential or simultaneous use of several, so that the limitations of each find compensation in the others. Here, nine techniques are deployed using one experimental rig, and compared to determine how accurately and rapidly they can characterize given bubble populations. These are, specifically (i) two stationary bubbles attached to a wire; and (ii) injected, rising bubbles. (C) 1997 Acoustical Society of America. [S0001-4966(97)00905-3]
\end{abstract}

PACS numbers: 43.30.Jx, 43.30.Pc, 43.25.Yw, 43.35.Ei [JHM]

\section{INTRODUCTION}

Bubble detection is required for many industrial, ${ }^{1}$ medical, ${ }^{2}$ and environmental ${ }^{3}$ applications. ${ }^{4,5}$ Throughout the range of acoustic techniques by which this can be achieved, there are inherent limitations. If, for example, a signal ${ }^{6,7}$ is capable of interpretation in terms of assigning homogeneous bulk properties to the "bubbly liquid" as a whole, then such interpretation may be limited to relatively high, relatively spatially uniform, bubble population densities, where the interbubble spacing is very much less than the acoustic wavelength. In contrast, other signals may be practicable only at low number densities. ${ }^{8,9}$ Several are prone to false triggering, in that some other object (e.g., a solid body, or a cluster of small bubbles) ${ }^{10}$ may give the same signal as that obtained from a given bubble.

In water with ambient pressure $p_{0}$, an air bubble of radius $R_{0}>\sim 10 \mu \mathrm{m}$ has a well-defined resonance frequency $f_{0}=\omega_{0} / 2 \pi \approx 0.01\left(\sqrt{p_{0}}\right) / R_{0}$, and pulsates as a lightly damped oscillator: On entrainment the pulsations generate an acoustic "signature," an exponentially decaying sinusoid, the frequency of which indicates the bubble size. ${ }^{11,12} \mathrm{~A}$ few milliseconds after entrainment these passive emissions have decayed to below the level of the noise. However, the bubble may still be driven, and active acoustic techniques exploit this acoustic resonance ${ }^{6,7,13,14}$ through measurements of sound speed, attenuation, scattering, etc. In such procedures, at a particular frequency the acoustic response of a bubbly liquid is taken to be dominated by bubbles which are resonant with that frequency. The maximum number of different bubble sizes that can be measured at any one time is determined by the number of different frequencies investigated, which historically is usually one, but in notable cases has been four ${ }^{13}$ or around nine. ${ }^{7}$ However, simple linear theory demonstrates that the acoustic scattering cross section of the fundamental frequency is only a local, and not a global, maximum at resonance: ${ }^{15}$ bubbles very much larger than resonance size can geometrically scatter sound to a greater degree than can smaller, resonant bubbles. If an ultrasonic interrogating signal is employed, the frequency of which is very much higher than the resonances of any bubbles in the sample, geometric scattering can detect bubbles. ${ }^{16-18}$ If $\mathrm{MHz}$ sound is, for example, employed to detect mm-sized bubbles, the small wavelengths involved $(\approx 0.4 \mathrm{~mm}$ in water at 3.5 $\mathrm{MHz}$ ) allow the bubble to be located, but do not accurately give the bubble size. Geometric, nonresonant scattering relies on the acoustic impedance mismatch between the inhomogeneity and the surrounding liquid. It is therefore insensitive to the nature of the inhomogeneity, and in practice may not distinguish between bubbles and solid bodies of a similar size.

A bubble in an acoustic pressure field $P=A \cos \omega_{p} t$ tends to linear, low-amplitude oscillations if the driving amplitude $A$ is small, or if the bubble is far from resonance. However, as the bubble pulsations become larger (for example, at resonance) the inherent nonlinearities in the motion become more pronounced, and manifest in the scattered acoustic signal as harmonics of the driving frequency. For example, a quadratic nonlinearity (i.e., a system response $\left.\propto P^{2}\right)$ will generate harmonics at $2 \omega_{p}$; higher-order nonlinearities give commensurate harmonics. This has been used to detect bubbles of specific size, resonant at $0.89 \mathrm{MHz}$ in one experiment ${ }^{19}$ and at both this and at $1.64 \mathrm{MHz}$ in another. ${ }^{20}$ If such systems are to be perfect bubble detectors then the condition must hold that only resonant bubbles can generate the required nonlinearity, and in the presence of only nonresonant bubbles, $\omega_{p}$ alone is detected. However, while the emission of the second harmonic is a global maximum at resonance, the $2 \omega_{p}$ signal can arise through nonbubble sources of nonlinearity, such as signal distortion in the equipment, which must be carefully examined. Such sources do not include solid inhomogeneities. The same condition holds if the applied field contains two frequencies, i.e., $P=A \cos \omega_{p} t+B \cos \omega_{i} t$ where $\omega_{p} \ll \omega_{i}$. The "imaging", frequency $\left(\omega_{i}\right)$ scatters geometrically from a target (the pulsating bubble) whose cross-sectional area varies periodically. ${ }^{9}$ The detected signal consists of $\omega_{i}$, modulated at frequency $\omega_{p}$, and the resulting detection of $\omega_{i} \pm \omega_{p}$ in the received spectrum has been used to size a bubble spectrum by employing the assumption that, bar the presence of resonant bubbles, only $\omega_{i}$ and $\omega_{p}$ are detected. ${ }^{8,21}$ The as- 


\begin{tabular}{|c|c|c|c|c|}
\hline Scatters & Advantage & Disadvantage & Prior application & $\begin{array}{l}\text { Bubble sizes } \\
\text { investigated in a } \\
\text { single expt. }\end{array}$ \\
\hline Geometric & $\begin{array}{l}\text { Rapidly obtains images } \\
\text { with high-spatial } \\
\text { (location) resolution. }\end{array}$ & Cannot distinguish between bubbles and solid particles. & Laboratory ${ }^{16,18,23}$ & $\begin{array}{l}\text { Distribution (low- } \\
\text { radius resolution) }\end{array}$ \\
\hline $\begin{array}{l}\text { Funda- } \\
\text { mental }\end{array}$ & Apparatus simple. & $\begin{array}{l}\text { Large bubbles and bubble clouds may falsely register as } \\
\text { resonant bubble (geometric scattering). Low-spatial } \\
\text { resolution. False triggering and off-resonance scattering } \\
\text { may occur. High-number densities only are valid if } \\
\text { "bulk properties"' are assigned to the liquid. }\end{array}$ & $\begin{array}{l}\text { Resonator }^{7,22} \\
\text { Attenuation }^{6} \\
\text { Backscatter }^{14}\end{array}$ & $\begin{array}{l}\text { Four; }{ }^{13} \text { around } \\
\text { nine }^{7,22}\end{array}$ \\
\hline $\begin{array}{l}\text { Second } \\
\text { harmonic }\end{array}$ & $\begin{array}{l}\text { Little contribution from } \\
\text { geometric scattering. }\end{array}$ & $\begin{array}{l}\text { Low-spatial resolution. False triggering and off-resonance } \\
\text { scattering may occur. }\end{array}$ & $\begin{array}{l}\text { Clinical, detecting } \approx \mu \mathrm{m} \\
\text { radius bubbles }\end{array}$ & $\begin{array}{l}\text { One }^{19} \text { or two } \\
\text { trial }\end{array}$ \\
\hline$\omega_{i} \pm \omega_{p}$ & No threshold. & False triggering and off-resonance scattering may occur. & Lab., ${ }^{8,21}$ field $^{24}$ & Distribution \\
\hline$\omega_{i} \pm \omega_{p} / 2$ & $\begin{array}{l}\text { Minimal false triggering } \\
\text { or, at threshold, } \\
\text { off-resonance scattering. }\end{array}$ & $\begin{array}{l}\text { Insonation at the threshold acoustic pressure is required for } \\
\text { fine radius resolution. }\end{array}$ & Laboratory ${ }^{9,25-27}$ & $\begin{array}{l}\text { One at } 25 \mathrm{~Hz} \\
\text { resolution }\end{array}$ \\
\hline
\end{tabular}

sumption fails if the pulsation of nonresonant bubbles, or the presence of a quadratic nonlinearity anywhere in the system (for example, through turbulent water motion), is sufficient to generate an $\omega_{i} \pm \omega_{p}$ signal. One advantage of combination-frequency methods is that the bubble resonance generates a signal in the $\mathrm{MHz}$ range (close to $\omega_{i}$ ), removing it from "masking" signals such as the acoustic input and ambient noise.

All the above techniques for bubble sizing which exploit the bubble resonance suffer in that sources other than resonant bubbles (e.g., turbulence, transducer effects, etc.) can to a greater or lesser extent generate the desired signal, indicating the presence of a resonant bubble when one is not present. ${ }^{4}$ Such "false triggering", has not to date been found when signals at $\omega_{i} \pm \omega_{p} / 2$ are used for bubble sizing. ${ }^{9}$ These signals are generated when the amplitude component $A$ of the insonating field $P=A \cos \omega_{p} t+B \cos \omega_{i} t$ exceeds the threshold value required to generate Faraday waves on the bubble surface. Characteristics of the various acoustic sizing techniques are summarized in Table I.

The less prone a system is to "false triggering," the more complicated in general it is to deploy. It therefore would be desirable to be able to deploy a range of these techniques to interrogate a given liquid sample, either sequentially or concurrently as defined by the problem. This would enable optimization of the process of characterizing the bubble population in the liquid with respect to minimizing the ambiguity of the result and the complexity of the task. The task itself involves first the detection of inhomogeneities in liquids. In certain circumstances it is then necessary to analyze the sample further to distinguish gas bubbles from solid or immiscible liquid-phase inclusions. The final stage of analysis would involve not only the detection, but also the sizing of the gas inclusions, leading to the characterization of the bubble population. This can be summarized in a four-part ideal objective: ${ }^{28}$ (i) Detect inhomogeneities in liquids; (ii) Distinguish gas bubbles from solids; (iii) Measure radii of bubbles present; (iv) Measure number of bubbles in each radius class.

This study introduces a method by which the ideal $o b$ jective might eventually be achieved, using a range of tech- niques. The limitations of each can be compensated through the deployment of others. Since the ambiguities of each have been studied theoretically and experimentally, ${ }^{15}$ the initial emphasis of this study will be how successfully each technique can provide information about simple controlled populations (stationary single and paired bubbles). A rising bubble stream will then be measured. The techniques listed in Table I are used, so that bubble detection is achieved through the geometric scattering of $3.5-\mathrm{MHz}$ ultrasound (using a scanner in both $\mathrm{B}$ and $\mathrm{M}$ modes simultaneously), and through scattering of signals at $\omega_{p}, 2 \omega_{p}, \omega_{p} / 2, \omega_{i} \pm \omega_{p}$, $\omega_{i} \pm 2 \omega_{p}, \omega_{i} \pm \omega_{p} / 2$, and $\omega_{i} \pm 3 \omega_{p} / 2$. This is done for broadband, and increasing, incremented, tonal "pump" signals. The study was carried out using relatively lowamplitude acoustic fields to drive the bubble, which is desirable to minimize the invasiveness of the technique.

\section{METHOD}

There exist detection zones, at $15-\mathrm{cm}$ depth, for the various active acoustic sizing systems (including those listed in Table I), comprising the overlap of beam patterns of relevant transducers held in rigid "cage"' configuration (Fig. 1). The cage is placed at depth $0.15 \mathrm{~m}$ in a $1.8 \times 1.2 \times 1.2-\mathrm{m}$ deep vibration isolated glass-reinforced plastic tank. The bubble population is either injected into the tank below these zones, and then rises to pass through them; or consists of bubbles attached to a wire, held within the intersection of the zones. A Gearing and Watson UW60 loudspeaker (having a frequency response flat to within $\pm 5 \mathrm{~dB}$ over the range 500 $\mathrm{Hz}-10 \mathrm{kHz}$ ) is used to generate the required "pump" signal. This signal drives the bubbles into oscillation, and it may be broadband, or a series of tones $P=A \cos \omega_{p} t$, where $\omega_{p}$ is incremented in $50 \mathrm{~Hz}$ (tethered bubbles) or $100 \mathrm{~Hz}$ (moving bubbles) steps.

During combination-frequency tests the imaging signal $P=B \cos \omega_{i} t$ is generated by a Therasonic 1030 (ElectroMedical Supplies) fixed at 1.134 MHz. A Panametrics V302 receiver detects the $\mathrm{MHz}$ signal before it is heterodyned with the Therasonic signal. The Bruel \& Kjaer 8103 hydrophone ("HP1') is used to detect signals not involving combination 


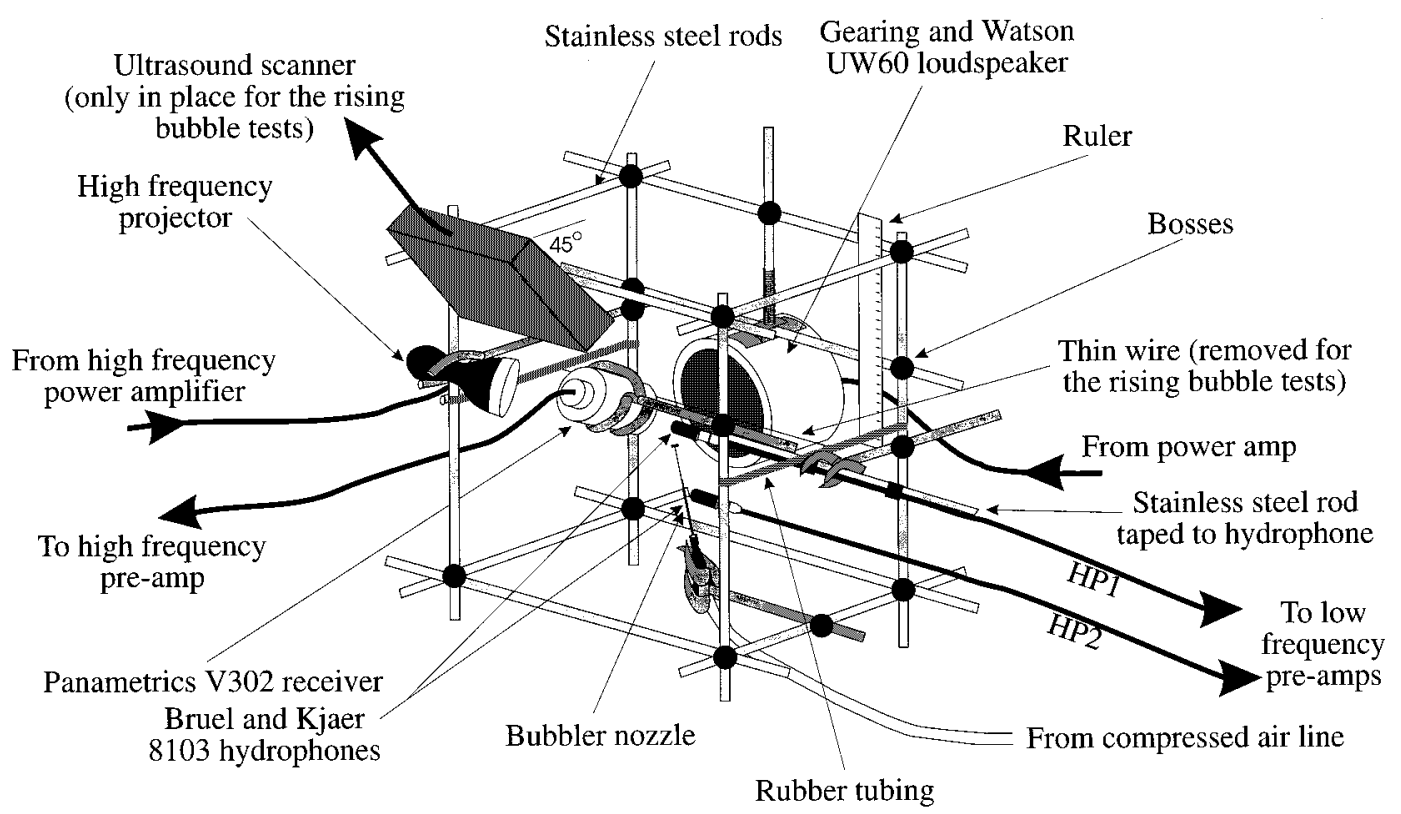

FIG. 1. Schematic of apparatus mounted in cage. For tethered bubble tests the ultrasound scanner is removed. For rising bubble tests the thin wire in the cage center is removed.

frequencies. The heterodyned high-frequency signal and the $\mathrm{B} \& \mathrm{~K} 8103$ signals are acquired to the PC via a general purpose interface bus (GPIB)-controlled Digital Storage Oscilloscope (LeCroy 9314L). Calibration is made with no bubbles present to allow compensation for the acoustic response of the water, apparatus, and tank. This enables the sample to be insonated at equal amplitudes when interrogated by a sequence of tonal pumping signals, each of $0.2-\mathrm{s}$ duration. Data is only collected after a "start-up" time of the first $7.5 \mathrm{~ms}$ for tethered bubbles, to allow transients to die away. No such delay can be afforded with rapidly rising $\mathrm{mm}$-sized bubbles, though averaging over the $10^{4}$ samples of each increment reduces the transient effect. Including data collection, the individual incremented tones start 1.6-s apart.

The rising bubbles are injected from a needle attached to a compressed air line. The passive acoustic signal so generated is detected by "HP2," a hydrophone (Bruel \& Kjaer 8103 ) placed $10 \mathrm{~mm}$ from the needle tip at a depth of $29 \mathrm{~cm}$. This signal is analyzed for the exponentially decaying sinusoid "signatures" which indicate the generation of each bubble, the frequency of the sinusoid revealing the bubble size. However, with higher entrainment rates (where signatures overlap) in noisy environments, individual entrainments may not be detected even in time-frequency representation (TFR), where resolution in time and frequency is a compromise determined by the size of the window imposed upon the data. However, a TFR of the Gabor coefficients, rather than the acoustic power invested in each frequency band, will readily identify the bubble signatures. ${ }^{29-31} \mathrm{~A}$ routine uses thresholds on the value and gradient of the Gabor coefficients, then automatically counts and sizes the bubbles, giving their rate of production before they rise into the active detection zones. A second count is made by placing a greased Petri dish in the rising bubble stream above the detection zones. Photographic measurement of the bubbles adhering to the thin layer of silicone grease were taken. How- ever, compensation must be made in comparing the bubble population measured in given volumes of liquid by the active techniques, with the captured population on the dish and the rate of bubble generation measured at the needle by the Gabor technique. This is because, for example, the volume of the bubble stream sampled by the Petri dish increases with the bubble rise speed. The volume changes caused by the varying hydrostatic pressure are accounted for in comparing all measurements. The sizes of the two bubbles attached to the wire were checked by detaching them from the wire into small glass flasks, in which they were transferred to a traveling microscope for measurement. ${ }^{25}$ A Hitachi EUB-26E 3.5-MHz ultrasound scanner, mounted in the cage, gave Mand B-mode images of the rising bubbles. Atmospheric pressure was $0.1003 \mathrm{MPa}$.

Detection through scattering at $\omega_{p}$ and $\omega_{i} \pm \omega_{p}$ requires only linear bubble pulsations, so that the relatively lowenergy densities per frequency band afforded by broadband insonation (bandlimited white noise between $1000-8000 \mathrm{~Hz}$ ) is appropriate. This rapidly allows an estimate of the region wherein the bubble resonances lie, for later application of the nonlinear detection signals $\left(2 \omega_{p}, \omega_{p} / 2, \omega_{i} \pm \omega_{p} / 2, \omega_{i}\right.$ $\left.\pm 2 \omega_{p}, \omega_{i} \pm 3 \omega_{p} / 2\right)$. These nonlinear signals require an incremented pure-tone pump signal, rather than broadband insonation, for two reasons. First, it is necessary to drive at a sufficiently high amplitude to generate detectable nonlinearities. Second, the detector frequency emitted by a bubble differs from that which drives it at resonance, which would cause ambiguity if broadband excitation were employed.

\section{RESULTS}

\section{A. Two tethered bubbles}

The first of the results are shown in Fig. 2 for the broadband excitation of two bubbles attached to a wire $10-\mathrm{mm}$ apart. Throughout the paper a dashed line indicates signal 

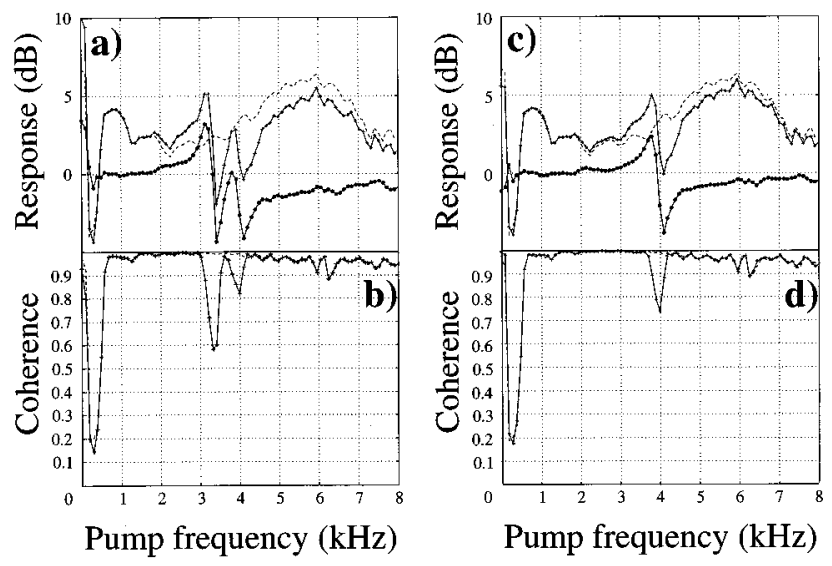

FIG. 2. Response [modulus of voltage transfer function, plots (a) and (c)] and coherence [(b) and (d)] for broadband insonation (band limited 1-8 $\mathrm{kHz}$ ) of both [(a) and (b)] tethered bubbles, and for just the smaller [(c) and (d)]: dashed line $=$ "in absence of bubbles"; $-+-+-=$ "in presence of bubbles"; - - = ratio of "bubble present" to "bubbles absent" signals. Resolution: $98 \mathrm{~Hz}$.

with no bubbles present; a solid line with crosses indicates the signal in presence of bubble(s); and a thick solid line with closed circles indicates the ratio of the signal "with" bubbles to that "without" bubbles, i.e., the bubble-mediated amplification. Data points occur at symbols, and at equivalent frequencies for dashed lines. Figure 2(a) illustrates the difference in the modulus of the voltage transfer function (the ratio of output to input) when the bubbles were driven by bandlimited $(1-8 \mathrm{kHz})$ white noise. The response shows peaks at 3.1 and $3.9 \mathrm{kHz}( \pm 0.1 \mathrm{kHz})$, with a sharp dip $\sim 300 \mathrm{~Hz}$ above each. This reflects the through-resonance behavior of each bubble: At frequencies just below resonance the sound field and the bubble pulsations (which scatter significantly more than they do away from resonance) are in phase and constructively interfere. However, above resonance the bubble undergoes a $\pi$ phase shift such that it now pulsates in antiphase with the driving sound field, resulting in destructive interference. This behavior suggests that the change in signal which results from bubble presence does not represent geometric scattering from a large bubble or other body, but is due to the presence of resonant bubbles in that frequency range. Even several $\mathrm{kHz}$ above the resonance of the pair, the detected signal is $\sim 1 \mathrm{~dB}$ less than the levels at low frequencies, and those found in the absence of bubbles. This is a result of the destructive interference caused by the whole population, and it may be that this can be used to characterize a population [compare this reduction with the smaller one seen in Fig. 2(c) for one of these bubbles on its own]. The coherence between the signal input to the source and the returned signal [Fig. 2(b)] shows a definite bubblemediated reduction in the signal around $3.3 \pm 0.15$ and 4 $\pm 0.15 \mathrm{kHz}$. As these coherence dips appear at frequencies midway between the peaks and troughs in the transfer function [Fig. 2(a)] they appear to indicate a bubble nonlinearity rather than a reduced signal to noise ratio, which would be the case if the dips in Fig. 2(a) and (b) occurred at the same frequency.

Figure 2(c) and (d) show the transfer function and co- herence resulting from broadband excitation when the bubble resonant at $\sim 3.3 \mathrm{kHz}$ is removed after completion of the two-bubble tests. Its peak disappears [Fig. 2(c)]. The other peak remains at $3.9 \pm 0.1 \mathrm{kHz}$, suggesting that to within this resolution the bubbles were far enough apart (approximately 10 bubble radii) for the bigger bubble not to significantly influence the resonance frequency of the other. ${ }^{32}$ The peak is about $3 \mathrm{~dB}$ higher than in the two bubble test even though the same excitation amplitude was used. This is due to the removal of the antiphase bubble pulsation of the larger bubble beyond its resonance, which therefore means that there is no destructively interfering component on the smaller bubble's pulsation below its resonance. The coherence again shows a similar dip to the relevant one found in the two bubble test [Fig. 2(d)].

Having, through $1 \mathrm{~s}$ (5 averages) of broadband insonation of the two bubbles, reduced the range of interest for further investigation from $1-8 \mathrm{kHz}$ to $2.7-4.7 \mathrm{kHz}$, the bubble pair was excited (with pump amplitude $120 \mathrm{~Pa}$ ) at 40 discrete increasing frequencies in $50-\mathrm{Hz}$ increments: At $1.6 \mathrm{~s}$ per increment, the test took $64 \mathrm{~s}$. The results are given in Fig. 3 for the harmonic [parts (a)-(c)] and sum-and-difference [parts (d)-(f)] signals, monitored simultaneously. The data is displayed as the magnitude in the frequency domain at the location of the signal of interest (i.e., at $\omega_{p}, 2 \omega_{p}, \omega_{p} / 2$, $\omega_{i} \pm \omega_{p}, \omega_{i} \pm 2 \omega_{p}$, and $\left.\omega_{i} \pm \omega_{p} / 2\right)$ corresponding to each pump frequency. The data was sampled at $50 \mathrm{kHz}$, and the FFT frequency resolution with 8192 points was $6 \mathrm{~Hz}$. The test was repeated following the removal of the larger bubble (Fig. 4).

The fundamental backscatter [Fig. 3(a)] shows a rippled amplitude response in the absence of a bubble, which is due to the differences in the proximity of each pumping signal tone to an FFT bin center frequency. This effect disappears when the $\mathrm{dB}$ difference ("amplification") between the signal with, and without, bubbles is taken, revealing again the characteristic through-resonance response indicating the presence of resonant bubbles at $3325 \pm 70$ and $3900 \pm 100 \mathrm{~Hz}$. The response of the second harmonic [Fig. 3(b)] is less clear. The height of the signal in the absence of the bubble can be affected for instance by the relative levels of harmonic distortion in the equipment and also the proximity of the signal to a frequency bin. Nevertheless, there still appears to be a clear increase in the signal between 3200-3400 and 3800$4100 \mathrm{~Hz}$. Removal of the larger bubble has negligible effect in the peaks in the first harmonic and second harmonic response for the smaller bubble as shown in Fig. 4(a) and (b). The emissions of $\omega_{p} / 2$ from both two bubbles [Fig. 3(c)] and the smaller one [Fig. 4(c)] are too small to differentiate from the noise floor. The amplitude of the heterodyned returned signal from the high-frequency receiver at $\omega_{i} \pm \omega_{p}, \omega_{i}$ $\pm 2 \omega_{p}$, and $\omega_{i} \pm \omega_{p} / 2$ are shown in Fig. 3(d)-(f) as a function of the incrementing pumping frequency $\omega_{p}$. Though there are maxima at $3.25 \pm 0.05$ and $3.9 \pm 0.2 \mathrm{kHz}$, the signal at $\omega_{i} \pm \omega_{p}$ [Fig. 3(d)] is present at more than $12 \mathrm{~dB}$ above the "no bubble" signal over the entire pumping frequency range. Clearly, the off-resonance contribution to the returned signal limits the resolution of the measurement for the bubble's resonance frequency. Though the off-resonance contri- 


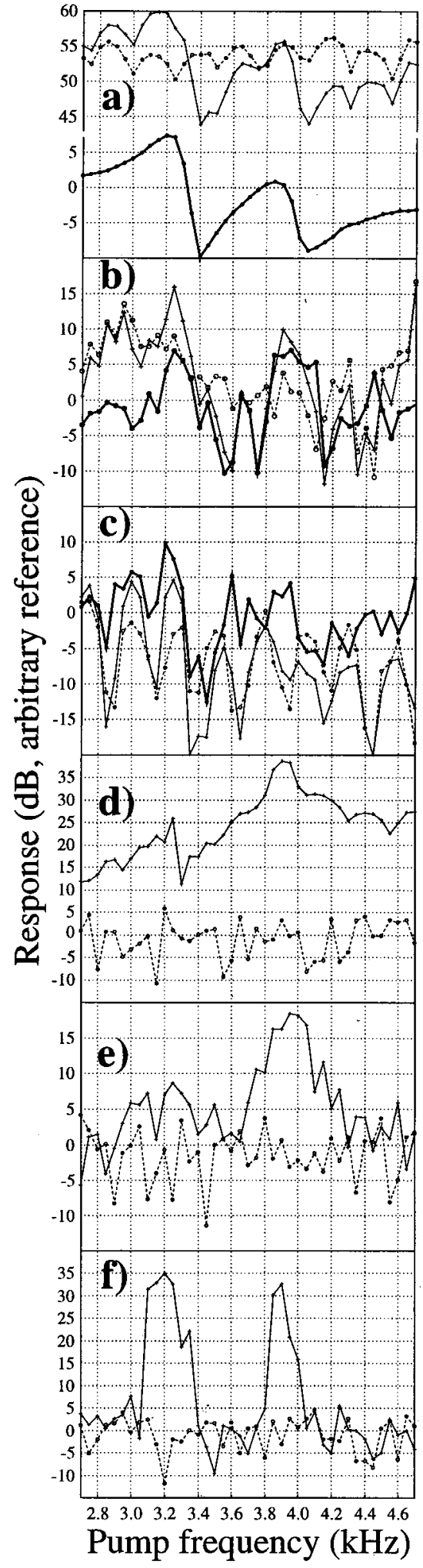

FIG. 3. The HP1 signals for the two-bubble tests $(50-\mathrm{Hz}$ increments) showing (a) $\omega_{p}$, (b) $2 \omega_{p}$, (c) $\omega_{p} / 2$, (d) $\omega_{i} \pm \omega_{p}$, (e) $\omega_{i} \pm 2 \omega_{p}$, (f) $\omega_{i} \pm \omega_{p} / 2$. Key as for Fig. 2, with open circles showing data points on dashed line.

bution is less for $\omega_{i} \pm 2 \omega_{p}$ [Fig. 3(e)] the resolution of the high-frequency peak is similarly poor $(4 \pm 0.2 \mathrm{kHz})$, and there are spurious maxima. It is clear that the $\omega_{i} \pm \omega_{p} / 2$ [Fig. 3(f)] signal best shows the presence of two bubbles, resonat-

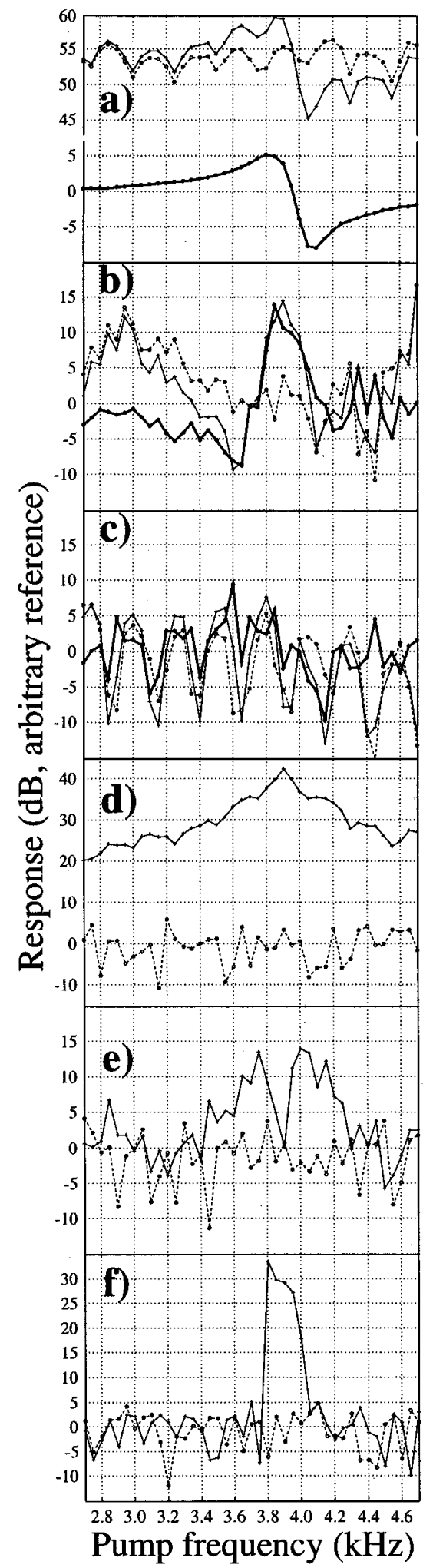

FIG. 4. As for Fig. 3, but for the smaller bubble only. Key as for Fig. 3.

ing at $3.2 \pm 0.1$ and $3.88 \pm 0.05 \mathrm{kHz}$. The off-resonance contributions are negligible. Removal of the larger bubble demonstrates the same features in the detection of the remaining bubble (Fig. 4) by the (d) $\omega_{i} \pm \omega_{p}$, (e) $\omega_{i} \pm 2 \omega_{p}$, and (f) $\omega_{i} \pm \omega_{p} / 2$ signals. 

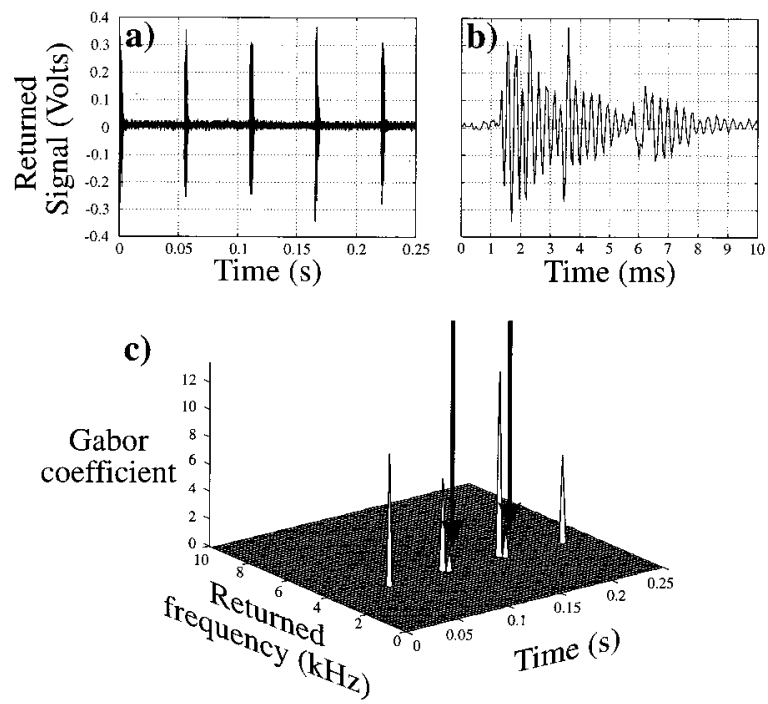

FIG. 5. The HP2 signal during injection. (a) Time series [detail shown in (b)]. (c) Time-frequency representation of Gabor coefficients associated with (a) (first peak removed for clarity). Where multiple coefficients are identified with injection of a single bubble, the later one (arrowed) gives natural frequency.

\section{B. Rising bubbles}

Figure 5 shows a portion of the bubble stream as measured through the passive acoustic emissions generated on injection. In Fig. 5(a) a 0.25-s section of the time series recorded by the hydrophone HP2 indicates individual bubbles being repeatably generated every $\sim 0.06 \mathrm{~s}$. Each of the bubble signatures has the form, not of a single exponentially decaying transient, but of multiple ones, revealing that the released bubble is excited on three subsequent occasions following the initial release from the needle [Fig. 5(b)]. These excitations arise through contact, and usually coalescence, between the newly released bubble and the successor gas pocket growing at the nozzle tip. ${ }^{33}$ As a result, the plot of the Gabor coefficients [Fig. 5(c)] may reveal multiple peaks for a single bubble (which vary each time, showing the nozzle process is not entirely repeatable). Clearly the frequency at which the final peak of each group occurs [arrowed in Fig. 5(c)] is the one which relates to the size of the final bubble after it has escaped clear of the contact/ coalescent processes that occur at the nozzle. It is this size which is taken to be a measure of the bubble size upon injection.

In Fig. 6 the results of broadband insonation in the frequency range $1-8 \mathrm{kHz}$ is shown. In Fig. 6(a), the signal picked up by HP1 is shown, both for the situation before the bubble stream began, and for the scattered signal in the presence of the bubble stream. The difference between the two signals is plotted, showing significant changes in the frequency range $3.5-5 \mathrm{kHz}$, indicating the through-resonance effect described above, centered around $4 \pm 0.1 \mathrm{kHz}$. In Fig. $6(\mathrm{~b})$, the heterodyned signal from the high-frequency receiver transducer shows bubble-mediated change from 3.5 to $4.9 \mathrm{kHz}$ (centered at $4.2 \pm 0.3 \mathrm{kHz}$ ). An $800-\mathrm{Hz}$ high-pass filter was placed after the heterodyning so that the strong Doppler components of the returned signal did not overload the input channel to the oscilloscope.
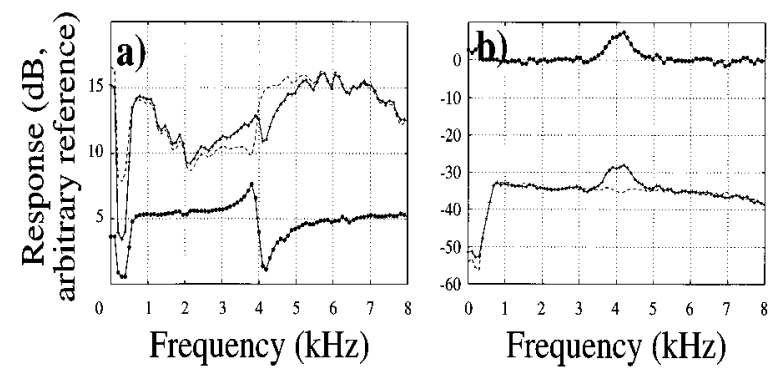

FIG. 6. Response (modulus of voltage transfer) for broadband insonation (bandlimited 1-8 kHz) of rising bubbles, from (a) HP1, and (b) heterodyned high-frequency (from V302) signals. Resolution: 98 Hz. Key as for Fig. 2.

Having rapidly found the region of interest (3.3-4.3 $\mathrm{kHz}$ ) through the broadband technique, the pump sound field is incremented in this range in steps of $100 \mathrm{~Hz}$, at a pressure amplitude of $240 \mathrm{~Pa}(0-\mathrm{pk})$. Figure 7 shows the results of analysis of the signal recorded by hydrophone HP1. In Fig. $7(\mathrm{a})$, the scattering of the fundamental frequency $\omega_{p}$ gives $f_{0} \approx 3850 \pm 20 \mathrm{~Hz}$. The second harmonic $2 \omega_{p}$ neither immediately indicates a distribution around a single bubble size [Fig. 7(b)], nor accurately indicates what the size might be $\left(f_{0} \approx 3.9 \pm 0.2 \mathrm{kHz}\right)$. The $\omega_{p} / 2$ results are similarly unclear [Fig. 7(c)]. During the same single pass from 3.2 to $4.4 \mathrm{kHz}$ as was made for Fig. 7, were taken the results for Fig. 8, a histogram showing the received, heterodyned spectrum as a function of the pump frequency (this, on the horizontal axis,

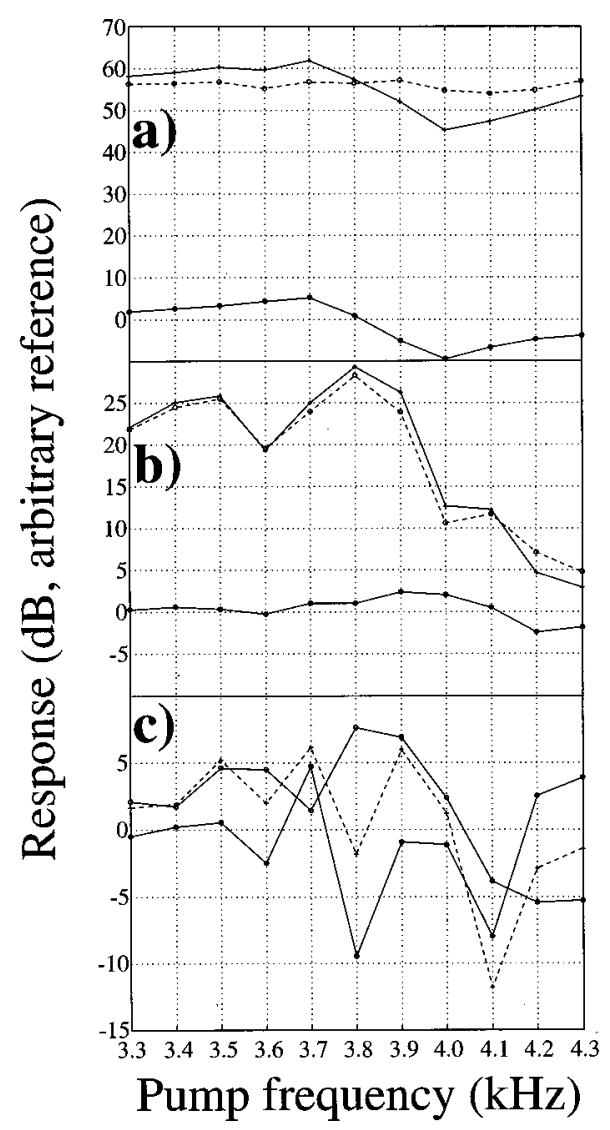

FIG. 7. Response at (a) $\omega_{p}$, (b) $2 \omega_{p}$, (c) $\omega_{p} / 2$ in the HP1 signal for insonation in $100-\mathrm{Hz}$ increments. Key as for Fig. 3. 


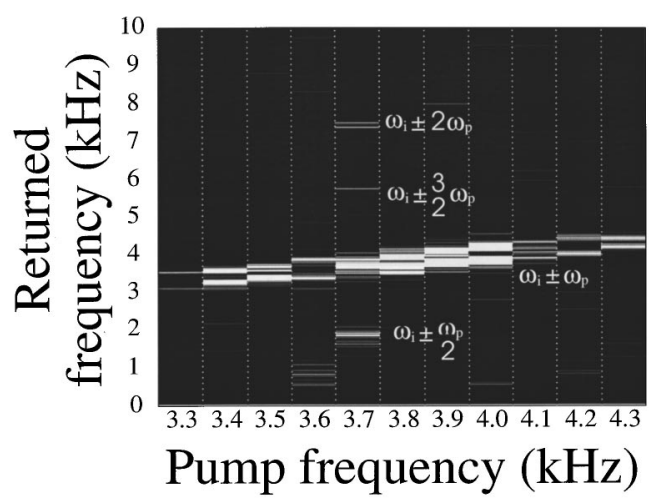

FIG. 8. Greyscale histogram showing heterodyned received signal (from V302) for each discrete setting of the pump frequency (100-Hz increments). Light shades indicate strong signal. Signals at $\omega_{i} \pm \omega_{p} / 2, \omega_{i} \pm \omega_{p}, \omega_{i}$ $\pm 3 \omega_{p} / 2$, and $\omega_{i} \pm 2 \omega_{p}$ are indicated.

indicating not a continuum but the 12 settings of the pump frequency, since the latter was incremented in $100-\mathrm{Hz}$ steps).

The clearest indication of resonance is that only for the pump frequency setting of $3.7 \mathrm{kHz}$ does structure in the heterodyned spectrum at frequencies which are multiples of $\omega_{p} / 2$ (corresponding to $\omega_{p} / 2, \omega_{p}, 3 \omega_{p} / 2$, and $2 \omega_{p}$ ) occur. All other peaks do not correspond to multiples of $\omega_{p}$. Figure 9 shows both the (a) M- and (b) B-mode images obtained using the Hitachi ultrasound scanner, the section shown being a slice at $45^{\circ}$ to vertical (Fig. 1). The bubble (labeled B) can be located in Fig. 9(b) (near field is at top of image), which also images the loudspeaker (S) and part of the cage. The images which intersect the vertical line (L) in $1 \mathrm{~s}$ are plotted in Fig. 9(a): Almost 19 bubbles pass through the beam in that time, with rise speed (from the image, within the limits of the rectilinear bubble motion, adjusting for the

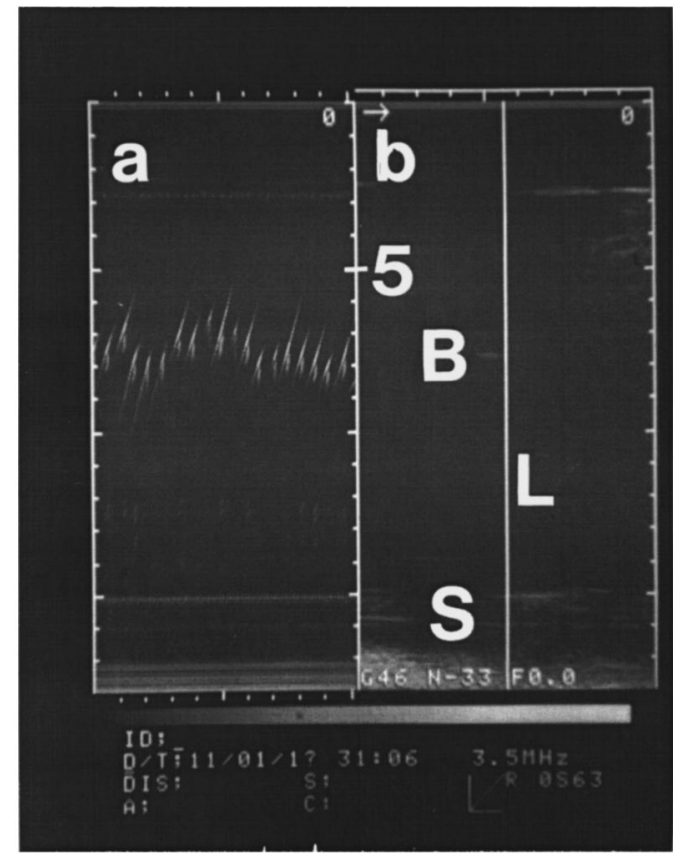

FIG. 9. (a) M-mode (1-s sweep) and (b) B-mode images from Hitachi ultrasound scanner. In (b) a bubble (B), UW60 speaker (S), the 5-cm marker from transducer faceplate (at top of image) and the line (L, occurrence of a target in which defines the M-mode image) are indicated. $45^{\circ}$ orientation) of $20 \pm 2 \mathrm{~cm} / \mathrm{s}$. Comparison of " a'" with " "b", allows the transient features (e.g., bubbles) to be distinguished from the time-invariant ones (e.g., cage and speaker).

\section{DISCUSSION}

For the two tethered bubbles, optical measurements gave radius estimates of $1.1 \pm 0.1$ and $0.8 \pm 0.1 \mathrm{~mm}$. Figure $3(\mathrm{f})$, which plots $\omega_{i} \pm \omega_{p} / 2$, most clearly indicates the presence of two bubbles. Table II summarizes the information gleaned from each signal type in the two-bubble test. Though no high-resolution technique determines both resonances to the same accuracy, the best overall resolution is obtained from $\omega_{i} \pm \omega_{p} / 2$ using incremented pump signals. Initial use of broadband first reduced the test time by a factor of 64 . The resolution of $\omega_{i} \pm \omega_{p} / 2$ can be dramatically affected by the acoustic pressure at the bubble: While it could be improved to $\pm 12 \mathrm{~Hz}$ by insonating at the threshold pressure, ${ }^{9}$ there is no guarantee that in the general case this threshold can be accurately delivered.

This is particularly true when considering the results from moving bubbles (Table III), since each bubble is transitory. Also because of this, not only the accuracy but also the population sampling must be considered. In fact, the results in Table III refer to two quite separate populations. First the incremented techniques (while they can be repeated to average a steady-state population $)^{9}$ were here in fact applied in one pass, and so would ideally detect signals only from resonant bubbles which are in the detection zone during the $0.2 \mathrm{~s}$ of each tone. Since bubbles are generated at $\sim 60 \mathrm{~ms}$ intervals, and have a rise time of $20 \pm 2 \mathrm{~cm} / \mathrm{s}$, all the incremented tests (columns 4-10) sample in each increment the same population of $\sim 4$ bubbles (different sets of $\sim 4$ bubbles for each of the 40 increments- "population 1"). Three minutes later the broadband techniques sample across the entire frequency range for five 0.2 -s averages, totalling 1 s: The results in columns 2 and 3 therefore sample a population of $\sim 19$ bubbles ("population 2"). Though there are differences in resolution between the broadband and the incremented techniques, the results in Table III indicate that the two populations differed, the one measured first having a lower resonance $(3.7 \pm 0.05 \mathrm{kHz})$ than the other $(4.0 \pm 0.1$ $\mathrm{kHz})$. This issue will be discussed later.

Resolution of the $\omega_{p}$ and $\omega_{i} \pm \omega_{p}$ signals is roughly constant between broadband and incremented forcing at around 100 and 300-500 Hz, respectively (Table III). The $\omega_{p}$ signal is not pronounced and would readily be confused by a wide range of sizes (see Table II). The resonance is indicated not by the maximum (strong emission almost in phase with driver), but by the in-phase point between the maximum and the minimum (antiphase) point: This has implications for studies where the scattering is assumed to be from resonant bubbles only. Only the simultaneous occurrence of the structure at $\omega_{i} \pm \omega_{p} / 2, \omega_{i} \pm 3 \omega_{p} / 2$, and $\omega_{i} \pm 2 \omega_{p}$ allows accurate active characterization. It is not surprising that the $\omega_{i}$ $\pm \omega_{p} / 2$ signal should so clearly indicate the resonance, 
TABLE II. Resonances and calculated radii of the two tethered bubbles $\left(p_{0}=101770 \mathrm{~Pa}\right)$. References in row 2 are to figures.

\begin{tabular}{|c|c|c|c|c|c|c|c|c|}
\hline & \multicolumn{2}{|c|}{$\longleftrightarrow-$ Broadband pump signal $\longrightarrow$} & \multicolumn{6}{|c|}{$\longleftrightarrow-$ Pump signal $(50 \mathrm{~Hz}$ increments, frequency increasing) $\longrightarrow$} \\
\hline & Amplitude $\omega_{p}$ & Coherence $\omega_{p}$ & $\omega_{p}$ & $2 \omega_{p}$ & $\omega_{p} / 2$ & $\omega_{i} \pm \omega_{p}$ & $\omega_{i} \pm 2 \omega_{p}$ & $\omega_{i} \pm \omega_{p} / 2$ \\
\hline $\begin{array}{l}\text { Distribution } \\
\text { indicated }\end{array}$ & $\begin{array}{l}\text { (2a) Two } \\
\text { bubbles }\end{array}$ & $\begin{array}{l}\text { (2b) Two } \\
\text { bubbles }\end{array}$ & $\begin{array}{l}\text { (3a) Two } \\
\text { bubbles }\end{array}$ & $\begin{array}{c}\text { Spurious } \\
\text { peaks (3b) }\end{array}$ & $\begin{array}{c}\text { Spurious } \\
\text { peaks (3c) }\end{array}$ & $\begin{array}{l}\text { (3d) Two } \\
\text { bubbles }\end{array}$ & $\begin{array}{c}\text { Unclear } \\
\text { peaks (3e) }\end{array}$ & $\begin{array}{l}\text { (3f) Two } \\
\text { bubbles }\end{array}$ \\
\hline Resonance freq. & $3.3 \pm 0.1$ & $3.3 \pm 0.15$ & $3.33 \pm 0.07$ & $3.3 \pm 0.1$ & $3.2 \pm 0.1$ & $3.25 \pm 0.05$ & $\cdots$ & $3.2 \pm 0.1$ \\
\hline$f_{0} / \mathrm{kHz}$ & $4.0 \pm 0.2$ & $4.0 \pm 0.15$ & $3.9 \pm 0.1$ & $3.95 \pm 0.15$ & $\cdots$ & $3.9 \pm 0.2$ & $4 \pm 0.2$ & $3.88 \pm 0.05$ \\
\hline$R_{0} \quad \mathrm{p}_{0}^{\mathrm{I} / 2}$ & $970 \pm 30$ & $970 \pm 44$ & $960 \pm 20$ & $970 \pm 30$ & $1000 \pm 30$ & $980 \pm 15$ & $\ldots$ & $1000 \pm 30$ \\
\hline$\overline{\mu \mathrm{m}} \approx \overline{100 \mathrm{f}_{0}}$ & $800 \pm 40$ & $800 \pm 30$ & $820 \pm 20$ & $810 \pm 30$ & $\ldots$ & $820 \pm 42$ & $800 \pm 40$ & $820 \pm 10$ \\
\hline
\end{tabular}

whereas the $\omega_{p} / 2$ signal does not, since the surface activity that generates the subharmonic emission cannot itself propagate to distance as it does not involve any bubble volume changes. However, as these Faraday waves change the effective area presented to the imaging beam, they can cause a modulation in the scattered signal, and this signal will propagate to distance.

The question of whether the two populations, measured by broadband and incremented techniques, could possess the distribution difference suggested above must be addressed by reference to the other techniques used for determining the bubble size some minutes after the conclusion of the broadband tests. The $\pm 2-\mathrm{cm} / \mathrm{s}$ standard deviation on the $20-\mathrm{cm} / \mathrm{s}$ rise time translates ${ }^{34}$ to estimated lower and upper limits for radius in this water of 0.87 and $1.13 \mathrm{~mm}$. Clearly this is not sufficiently discerning. The distribution of rising bubbles from four Petri dish photographs (taken 10 minutes after the end of the passive Gabor tests and corrected for hydrostatic head) gives for the size at $15-\mathrm{cm}$ depth: $790 \pm 60 \mu \mathrm{m}(28$ bubbles collected in $1.5 \mathrm{~s}) ; 790 \pm 120 \mu \mathrm{m}$ ( 24 bubbles in 1.3 s); $830 \pm 80 \mu \mathrm{m}$ ( 27 bubbles in $1.4 \mathrm{~s}) ; 820 \pm 130 \mu \mathrm{m}$ (32 bubbles in $1.7 \mathrm{~s}$ ). There is some indication of occasional larger bubbles in a more uniform distribution.

The actual stability of the population is best determined by the Gabor tests. Three of these were performed at oneminute intervals after the broadband tests, and before the ultrasonic images were taken. In each test $0.25 \mathrm{~s}$ of passive emissions, comprising the injection emissions of five consecutive bubbles, were taken [Fig. 5(a) represents test 2]. The natural frequencies so found are shown in Table IV, with the average for each test, and the calculated bubble size distri- bution at the needle (29-cm depth) and at the zone of the active detector (15-cm depth). Clearly, variation in the size of the generated bubbles can occur. This is not unexpected when compressed air, supplied from a line, is bubbled at rates high enough for interbubble contact/coalescence to occur. Table IV suggests that the variation found during the $1 \mathrm{~s}$ of the broadband test, and the $40 \times 1.6 \mathrm{~s}$ of the incremented test, is of the same order as the standard deviations quoted in Table III. Clearly for all but the technique with the highest resolution in each population, the standard deviation must represents the resolution limitations of the techniques. For the highest resolution (columns $8-10$ for population 1; column 2 for population 2) the uncertainties in Table III are similar to those quoted for these techniques during the twobubble test (Table II), when the population was stable. This suggests that, here too, the standard deviations reflect limits in resolution. It seems that in fact the best resolution limits in each case are very similar to the variability one might expect in the population. Though by no way conclusive, it is suggestive that the large standard deviations in tests 1 and 3 result from single outlying values. These values could well escape detection in the 0.2-s duration of each incremented tone, and if the item $3190 \mathrm{~Hz}$ is removed from test 1 the average becomes $3686 \pm 90 \mathrm{~Hz}(871 \pm 21$ and $875 \pm 21 \mu \mathrm{m}$ at 29- and 15-cm depth, respectively), and if the item $3219 \mathrm{~Hz}$ is eliminated from test 3 , the average becomes $4004 \pm 30 \mathrm{~Hz}$ (giving $802 \pm 6$ and $806 \pm 6 \mu \mathrm{m}$ at $29-$ and $15-\mathrm{cm}$ depth, respectively). This variation is less than the resolution limits of Table II and the uncertainties quoted in Table III, for the $\omega_{i} \pm \omega_{p} / 2$ and related tests.

The Gabor technique for sizing bubbles from their pas-

TABLE III. Resonances and calculated radii of rising bubbles for populations 2 (broadband pump) and 1 (incremented pump).

\begin{tabular}{|c|c|c|c|c|c|c|c|c|c|}
\hline Column 1 & Column 2 & Column 3 & Column 4 & Column 5 & Column 6 & Column 7 & Column 8 & Column 9 & Column 10 \\
\hline $\begin{array}{l}\text { Distribution } \\
\text { indicated }\end{array}$ & $\begin{array}{c}\text { narrow } \\
\text { [Fig. 6(a)] }\end{array}$ & $\begin{array}{c}\text { broad } \\
\text { [Fig. 6(b)] }\end{array}$ & $\begin{array}{c}\text { narrow } \\
\text { [Fig. 7(a) }]\end{array}$ & $\begin{array}{l}\text { bimodal } \\
\text { [Fig. 7(b)] }\end{array}$ & $\begin{array}{l}\text { bimodal } \\
\text { [Fig. 7(c)] }\end{array}$ & $\begin{array}{c}\text { broad } \\
\text { (Fig. 8) }\end{array}$ & $\begin{array}{l}\text { narrow } \\
\text { (Fig. 8) }\end{array}$ & $\begin{array}{l}\text { narrow } \\
\text { (Fig. 8) }\end{array}$ & $\begin{array}{l}\text { narrow } \\
\text { (Fig. 8) }\end{array}$ \\
\hline$\frac{R_{0}}{\mu \mathrm{m}} \approx \frac{p_{0}^{1 / 2}}{100 f_{0}}$ & 800 & 760 & 830 & 840 & 820 & 840 & 862 & 862 & 862 \\
\hline$\mu \mathrm{m} \quad 100 \mathrm{f}_{0}$ & \pm 20 & \pm 50 & \pm 22 & \pm 33 & \pm 42 & \pm 110 & \pm 12 & \pm 12 & \pm 12 \\
\hline
\end{tabular}


TABLE IV. Natural frequencies and calculated average radii from Gabor tests at 29 , and $15-\mathrm{cm}$ depths.

\begin{tabular}{crrr}
\hline \hline Trial & Test 1 & Test 2 & Test 3 \\
\hline Natural frequencies/Hz & 3722 & 3751 & 4018 \\
& 3737 & 3699 & 4015 \\
& 3190 & 3642 & 3219 \\
& 3550 & 3758 & 3965 \\
Average freq./Hz & 3736 & 3835 & 4021 \\
& 3580 & 3740 & 3850 \\
$R_{0} / \mu \mathrm{m}$ at $29 \mathrm{~cm}$ & \pm 240 & \pm 70 & \pm 350 \\
& 897 & 859 & 834 \\
$R_{0} / \mu \mathrm{m}$ at $15 \mathrm{~cm}$ & \pm 60 & \pm 15 & \pm 76 \\
& 901 & 863 & 838 \\
& \pm 60 & \pm 16 & \pm 75 \\
\hline \hline
\end{tabular}

sive ringing upon formation is not only the most simple and accurate but also samples the entire population, being capable of logging the natural frequency of each and every bubble that is generated in near real time to $1 \mathrm{~Hz}$ accuracy (even giving details of nozzle processes). However, the Gabor signal must be interpreted carefully. It reflects the natural frequency of a damped system, given by $\omega_{0}\left(1-\delta^{2}\right)$, where $\delta$ is the dimensionless damping coefficient ${ }^{35}$ and $\omega_{0}$ the undamped natural frequency: Active techniques in general measure the maximum of the amplitude response, which occurs at frequency $\omega_{0}\left(1-2 \delta^{2}\right)$. The two major limitations of the Gabor technique are, first, that the signal becomes increasingly difficult to interpret as the entrainment rate increases. Second, passive emissions usually give information only about the bubbles being entrained during the measurement interval, the excitation that is strong enough to make adequate emissions usually requiring the closure of a liquid surface: ${ }^{15}$ Older, "silent" bubbles would have to be excited by impulse to ring, and a sufficiently strong impulse would alter the bubble population by inducing more closures (i.e., fragmentation).

\section{CONCLUSIONS}

Broadband insonation rapidly indicates the range over which bubble resonances may occur, reducing the time required for tonal incrementation. Best resolution and population sampling was achieved using the Gabor technique, though this operates only on entrainment. The best active indicator of the bubble population in these tests, where a relatively low-amplitude pump signal was employed to minimize the invasiveness of the technique, ${ }^{36}$ was the $\omega_{i} \pm \omega_{p} / 2$ signal. However, it must be remembered that this signal is not simple to implement: For best resolution the acoustic pressure amplitude at the bubble must be close to the threshold, ${ }^{9}$ and a delay (after insonation at a given frequency commences) is recommended, to allow the transients to decay before data is acquired.

\section{ACKNOWLEDGMENTS}

Our thanks to EPSRC (Reference No. GR/H 79815) and NERC (GR3/9992) for funding, and to P. R. White for advice.
${ }^{1}$ R. M. Detsch and R. N. Sharma, "The critical angle for gas bubble entrainment by plunging liquid jet,' Chem. Eng. J. 44, 157-66 (1990).

${ }^{2}$ E. G. Tickner, "Precision microbubbles for right side intercardiac pressure and flow measurements," in Contrast Echocardiography, edited by R. S. Meltzer and J. Roeland (Nijhoff, London, 1982).

${ }^{3}$ D. K. Woolf, "Bubbles and the air-sea transfer velocity of gases," Atmos.-Ocean 31, 451-474 (1993).

${ }^{4}$ T. G. Leighton, "Acoustic Bubble Detection. I. The detection of stable gas bodies," Environ. Eng. 7, 9-16 (1994).

${ }^{5}$ T. G. Leighton, “Acoustic Bubble Detection. II. The detection of transient cavitation," Environ. Eng. 8, 16-25 (1995).

${ }^{6} \mathrm{H}$. Medwin, "In situ acoustic measurements of microbubbles at sea," J. Geophys. Res. 82, 971-976 (1977).

${ }^{7}$ H. Medwin and N. D. Breitz, "Ambient and transient bubble spectral densities in quiescent seas and under spilling breakers," J. Geophys. Res. 94, 12751-12759 (1989)

${ }^{8}$ V. L. Newhouse and P. M. Shankar, "Bubble size measurement using the nonlinear mixing of two frequencies," J. Acoust. Soc. Am. 75, 14731477 (1984).

${ }^{9}$ A. D. Phelps and T. G. Leighton, "High resolution bubble sizing through detection of the subharmonic response with a two frequency excitation technique," J. Acoust. Soc. Am. 99, 1985-1992 (1996).

${ }^{10}$ R. Y. Nishi, "Ultrasonic detection of bubbles with Doppler flow transducers," Ultrasonics 10, 173-179 (1972).

${ }^{11}$ M. Strasberg, "'Gas bubbles as sources of sound in water," J. Acoust. Soc. Am. 28, 20-26 (1956).

${ }^{12}$ T. G. Leighton and A. J. Walton, “An experimental study of the sound emitted from gas bubbles in a liquid," Eur. J. Phys. 8, 98-104 (1987).

${ }^{13}$ D. M. Farmer and S. Vagle, "Waveguide propagation of ambient sound in the ocean-surface bubble layer," J. Acoust. Soc. Am. 86, 1897-1908 (1989).

${ }^{14}$ S. A. Thorpe, "Measurements with an Automatically Recording Inverted Echo Sounder; ARIES and the Bubble Clouds," J. Phys. Oceanogr. 16, 1462-1478 (1986).

${ }^{15}$ T. G. Leighton, The Acoustic Bubble (Academic, London, 1994), pp. 234243, 295-298, 439-464.

${ }^{16}$ S. L. Morriss and A. D. Hill, "Ultrasonic imaging and velocimetry in two-phase pipe flow," Trans. ASME, J. Heat Transf. 115, 108-116 (1993).

${ }^{17}$ R. Van Der Welle, "Void fraction, bubble velocity and bubble size in two phase flow," Int. J. Multiphase Flow 11, 317-45 (1985).

${ }^{18}$ W. F. Kolbe, B. T. Turko, and B. Leskovar, "Fast ultrasonic imaging in a liquid filled pipe," IEEE Trans. Plasma Sci. 33, 715-722 (1986).

${ }^{19}$ D. L. Miller, "Ultrasonic detection of resonant cavitation bubbles in a flow tube by their second harmonic emissions," Ultrasonics 19, 217-24 (1981).

${ }^{20}$ D. L. Miller, A. R. Williams, and D. R. Gross, "Characterization of cavitation in a flow-through exposure chamber by means of a resonant bubble detector," Ultrasonics 22, 224-230 (1984).

${ }^{21}$ R. M. Schmitt, H. J. Schmidt, B. Grohs, H. P. Schwarz, and M. Biebinger, "Bubble sizing in the lower micron range based on the 2-frequency insonification method," Ultrason. Imaging 9, 63-64 (1987).

${ }^{22} \mathrm{~N}$. Breitz and H. Medwin, "Instrumentation for in-situ acoustical measurements of bubble spectra under breaking waves," J. Acoust. Soc. Am. 86, 739-43 (1989).

${ }^{23}$ J. Wolf, "Investigation of bubbly flow by ultrasonic tomography," Part. Part. Syst. Charact. 5, 170-173 (1988).

${ }^{24}$ D. Koller, Y. Li, P. M. Shankar, and V. L. Newhouse, "High-speed bubble sizing using the double frequency technique for oceanographic applications,"' IEEE J. Oceanic Eng. 17, 288-291 (1992).

${ }^{25}$ T. G. Leighton, R. J. Lingard, A. J. Walton, and J. E. Field, “Acoustic bubble sizing by the combination of subharmonic emissions with an imaging frequency," Ultrasonics 29, 319-323 (1991).

${ }^{26}$ A. D. Phelps and T. G. Leighton, "Investigations into the use of two frequency excitation to accurately determine bubble sizes," in Bubble Dynamics and Interface Phenomena, Proceedings of an IUTAM Symposium, edited by J. R. Blake, J. M. Boulton-Stone, and N. H. Thomas (Kluwer Academic, Dordrecht, The Netherlands, 1994), pp. 475-483.

${ }^{27}$ A. D. Phelps and T. G. Leighton, "Acoustic bubble sizing using two frequency excitation techniques," Proceedings of the 2nd European Conference on Underwater Acoustics, Copenhagen, 1994, edited by L. Bjorno (European Commission, Luxenbourg, 1994), pp. 201-206.

${ }^{28}$ T. G. Leighton, A. D. Phelps, D. G. Ramble, and D. A. Sharpe, "Com- 
parison of the abilities of eight acoustic techniques to detect and size a single bubble," Ultrasonics 34, 661-667 (1996).

${ }^{29}$ T. G. Leighton, M. F. Schneider, and P. R. White, "Study of dimensions of bubble fragmentation using optical and acoustic techniques," Proceedings of the Sea Surface Sound, Lake Arrowhead, California, 1994, edited by M. J. Buckingham and J. Potter (World Scientific, Singapore, 1995), pp. 414-428.

${ }^{30}$ A. D. Phelps, T. G. Leighton, M. F. Schneider, and P. R. White, “Acoustic bubble sizing, using active and passive techniques to compare ambient and entrained populations," ISVR Technical Report No. 229, University of Southampton, UK, 1994.

${ }^{31}$ A. D. Phelps, T. G. Leighton, M. F. Schneider, and P. R. White, “Active and passive acoustic bubble sizing," ISVR Technical Report No. 237, University of Southampton, UK, 1994.
${ }^{32}$ M. Strasberg, "The pulsation frequency of nonspherical gas bubbles in liquids,'” J. Acoust. Soc. Am. 25, 536-537 (1953).

${ }^{33}$ T. G. Leighton, K. J. Fagan, and J. E. Field, “Acoustic and photographic studies of injected bubbles,” Eur. J. Phys. 12, 77-85 (1991).

${ }^{34}$ R. Clift, J. R. Grace, and M. E. Weber, Bubbles, Drops and Particles (Academic, New York, 1978).

${ }^{35}$ C. Devin, Jr., "Survey of Thermal, Radiation, and Viscous Damping of Pulsating Air Bubbles in Water," J. Acoust. Soc. Am. 31, 1654 (1959).

${ }^{36}$ T. G. Leighton, A. D. Phelps, and D. G. Ramble, "Bubble detection using low amplitude multiple acoustic techniques," Proceedings of the $3 \mathrm{rd} \mathrm{Eu-}$ ropean Conference on Underwater Acoustics, Heraklion, 1996, edited by J. S. Papadakis (Crete University, Heraklion, Crete, Greece), pp. 11431147. 